CARPATHIAN J. MATH.

Volume 37 (2021), No. 2,

Pages $235-258$
Online version at https : //www . carpathian. cunbm. utcluj. ro/

Print Edition: ISSN 1584 - 2851; Online Edition: ISSN 1843 - 4401

DOI: https://doi.org/10.37193/CJM.2021.02.10

Invited paper

\title{
Set-theoretical aspect of the fixed point theory: some examples
}

\author{
IOAN A. RUS
}

ABSTRACT. In this paper we revisit some of my own contributions to the fixed point theory, contributions which are related to set-theoretical terms. My intention is to do this in a unitary way. New notions and results are given. Open problems are also formulated.

\section{INTRODUCTION}

In the fixed point theory there are notions and problems which are set-theoretical (see [55], [74], [103], [106], [107], [109], [111], [121], [126], ...). If $X$ is a structured set (ordered set, group, ring, algebra, $L$-space, topological space, metric space, generalized metric space, Banach space, Hilbert space, ordered $L$-space, ...) and $f: X \rightarrow X$ is an operator, then these set-theoretical problems are studied in terms of these structures on $X$. Moreover there are some fixed point results in the theory of category ([80], [14], [77], [24], [146], [81], [59], [103], [108], ...).

In this paper we revisit some of my own contributions, in the fixed point theory, in relation to set-theoretical terms. We shall do this in a unitary way. New notions and results are given, and open problems are formulated. The structure of the paper is as follows:

1. Introduction

2. Set-theoretical fixed point results: $R$-contractions

3. Equivalent statements with the set-theoretical one

4. Invariant operator with respect to an operator: invariant partition of a set with respect to an operator

5. Fixed point structures on an abstract set

5.1. Examples of fixed point structures

5.2. Compatible pair with a fixed point structure

5.3. Closure operators and invariant subsets

5.4. Fixed point theorems in terms of a compatible pair: $\theta$-condensing operators

6. Closure operators and invariant subsets in terms of iterates: $(\theta, l)$-contractions

6.1. Invariant subsets

6.2. Operators with intersection property

Received: 19.01.2021. In revised form: 26.04.2021. Accepted: 26.04.2021

2010 Mathematics Subject Classification. 54H25, 47H10, 03E20, 54A20, 47H08, 47H09, 41A36, 65J15, 34G20, $45 \mathrm{~N} 05$.

Key words and phrases. invariant subset, orbit, closure operator, invariant partition, cyclic covering, invariant operator with respect to an operator, interpolation point, equivalent fixed point equations, admissible perturbation of an operator, operator with intersection property, fixed point structure, compatible pair with a fixed point structure, $\theta$-condensing operator, strong $\theta$-condensing operator, $(\theta, \varphi)$-contraction, strong $(\theta, \varphi)$-contraction, $R$-space, $R$-contraction, retraction, retractible operator, boundary condition, open problem, conjecture. 
6.3. Fixed point theorems in terms of an operator with intersection property: $(\theta, l)$ contractions

7. Cyclic covering of a set with respect to an operator

8. Equivalent fixed point equations

8.1. Admissible perturbations of an operator

8.2. Operator with a weakly Picard operator perturbation

9. Set retractions and fixed points of nonself operators

10. Multivalued operators

Throughout this paper we follow the notation and terminology in [121], [126] and [140].

Regarding the set and category theory see: [58], [80], [14], [25], [24], [69], [76], [81], [82], [108], [59], [70], ...

Regarding the basic fixed point theorems in sets and structured sets see: [55], [74], [140], [54], [63], [70], [73], [106], [121], [48], [103], [105], [107], [109], ..

\section{SET-THEORETICAl FIXED POINT RESUlts: $R$-CONTRACTIONS}

Before 1988 there were given some set-theoretical fixed point theorems (Chu-Diaz [36], Abian [1], [2], Wisniewski [159], Eilenberg [55], Lim [79], Rus [102], [103], [106], Deaconescu [40]). From these we mention the following two:

Abian-Wisniewski Theorem ([2], [159]). Let $X$ be a nonempty set and $f: X \rightarrow X$ be an operator. Then the following statements are equivalent:

(i) $F_{f}:=\{x \in X \mid f(x)=x\}=\emptyset$

(ii) there exists three mutually disjoint subsets such that:

(a) $X=X_{1} \cup X_{2} \cup X_{3}$;

(b) $X_{i} \cap f\left(X_{i}\right)=\emptyset$, for each $i \in\{1,2,3\}$.

Eilenberg Theorem ([55], p.19). Let $X$ be a nonempty set and let $\left(R_{n}\right)_{n \in \mathbb{N}}$ be a sequence of equivalence relations in $X$ such that:

(1) $X \times X=R_{0} \supset R_{1} \supset \ldots \supset R_{n} \supset \ldots$;

(2) $\bigcap_{n \in \mathbb{N}} R_{n}=\Delta(X):=\{(x, x) \mid x \in X\}$;

(3) if $\left(x_{n}\right)_{n \in \mathbb{N}}$ is a sequence in $X$ such that $\left(x_{n}, x_{n+1}\right) \in R_{n}$, for each $n \in \mathbb{N}$, then there exists an $x \in X$ such that $\left(x_{n}, x\right) \in R_{n}$, for all $n \in \mathbb{N}$.

If $f: X \rightarrow X$ is such that,

$$
n \in \mathbb{N},(x, y) \in R_{n} \Rightarrow(f(x), f(y)) \in R_{n+1},
$$

then $f$ has a unique fixed point $x^{*}$ and $\left(f^{n}(x), x^{*}\right) \in R_{n}$, for each $x \in X$ and $n \in \mathbb{N}$.

In our papers [115] and [116], we consider, in the Eilenberg Theorem, instead equivalence relations $R_{n}$, the symmetric relations and in [116] we give a new notion: $R$ contraction. In what follows, we revisit this notion.

Let $X$ be an abstract set and $R:=\left(R_{n}\right)_{n \in \mathbb{N}}$ be a sequence of symmetric binary relations in $X$. By definition $R$ is an admissible sequence iff the following conditions are satisfied:

$\left(C_{1}\right) X \times X=R_{0} \supset R_{1} \supset \ldots \supset R_{n} \supset \ldots ;$

$\left(C_{2}\right) \bigcap_{n \in \mathbb{N}} R_{n}=\Delta(X)$;

$\left(C_{3}\right)$ the following implication holds,

$$
\left(x_{n}\right)_{n \in \mathbb{N}} \subset X, x, y \in X,\left(x_{n}, x\right) \in R_{n},\left(x_{n}, y\right) \in R_{n}, n \in \mathbb{N} \Rightarrow x=y .
$$

By definition we call a pair $(X, R)$, where $R$ is an admissible sequence, an $R$-space.

Remark 2.1. From $\left(C_{2}\right)$ we have that, if $(X, R)$ is an $R$-space, then the binary relations $R_{n}$, are reflexive. 
Definition 2.1. A sequence $\left(x_{n}\right)_{n \in \mathbb{N}}$ in $(X, R)$ is convergent if there exists $x \in X$ such that $\left(x_{n}, x\right) \in R_{n}$, for all $n \in \mathbb{N}$.

From $\left(C_{3}\right)$ it follows that the limit of a convergent sequence is unique. We denote a convergent sequence $\left(x_{n}\right)_{n \in \mathbb{N}}$ with the limit $x$, by $x_{n} \stackrel{R}{\rightarrow} x$.

By $\left(C_{2}\right)$ it follows that, if $x_{n}=x$, for all $n \in \mathbb{N}$, then $x_{n} \stackrel{R}{\rightarrow} x$. Also, from Definition 2.1 and $\left(C_{1}\right)$, each subsequence of a convergent sequence is a convergent sequence with the same limit as $\left(x_{n}\right)_{n \in \mathbb{N}}$.

From the above considerations it follows that an $R$-space structure on a set $X$ induces an $L$-space structure of Fréchet type on $X$ (see [49], [69], [43], [122], [140], ...).

Definition 2.2. An $R$-space $(X, R)$ is complete if the following implication holds: $x_{n} \in X,\left(x_{n}, x_{n+p}\right) \in R_{n}$, for all $n, p \in \mathbb{N} \Rightarrow\left(x_{n}\right)_{n \in \mathbb{N}}$ is convergent.

Remark 2.2. Let $(X, R)$ be an $R$-space, where $R=\left(R_{n}\right)_{n \in \mathbb{N}}$ is a sequence of equivalence relations and $\left(x_{n}\right)_{n \in \mathbb{N}}$ be a sequence in $X$. The following statements are equivalent:

(1) $\left(x_{n}, x_{n+1}\right) \in R_{n}$, for all $n \in \mathbb{N}$;

(2) $\left(x_{n}, x_{n+p}\right) \in R_{n}$, for all $n, p \in \mathbb{N}$.

Definition 2.3. Let $(X, R)$ be an $R$-space. By definition an operator $f: X \rightarrow X$ is an $R$-contraction if the following implication holds:

$$
(x, y) \in R_{n} \Rightarrow(f(x), f(y)) \in R_{n+1} \text {, for all } n \in \mathbb{N} .
$$

In the above terminology our basic results in [116] take the following form.

Theorem 2.1. Let $(X, R)$ be a complete $R$-space and $f: X \rightarrow X$ be an $R$-contraction. Then we have that:

(i) $F_{f}=F_{f^{n}}=\left\{x^{*}\right\}$, for all $n \in \mathbb{N}^{*}=\mathbb{N} \backslash\{0\}$;

(ii) $f^{n}(x) \stackrel{R}{\rightarrow} x^{*}$, for all $x \in X$.

Theorem 2.2. Let $(X, R)$ be a complete $R$-space and $f: X \rightarrow X$ be an operator. We suppose that there exists $k \in \mathbb{N}^{*}$ such that $f^{k}$ is an $R$-contraction. Then:

(i) $F_{f}=F_{f^{n}}=\left\{x^{*}\right\}$, for all $n \in \mathbb{N}^{*}$;

(ii) $f^{n}(x) \stackrel{R}{\rightarrow} x^{*}$, for all $x \in X$.

Proof. From Theorem 2.1, $F_{f^{k}}=F_{f^{n k}}=\left\{x^{*}\right\}$, for all $n \in \mathbb{N}^{*}$ and $\left(f^{n k}(x), x^{*}\right) \in R_{n}$, for all $n \in \mathbb{N}^{*}$ and $x \in X$. Since $f^{k}$ has a unique fixed point, $x^{*}$, it follows that (see Chu-Diaz [36]), $F_{f}=\left\{x^{*}\right\}$. If we take in the relations, $\left(f^{n k}(x), x^{*}\right) \in R_{n}$, for all $x \in X$ and instead of $x$, the following, $f(x), \ldots, f^{k-1}(x)$, we have that $\left(f^{n}(x), x^{*}\right) \in R_{n}$, for all $n \in \mathbb{N}$ and all $x \in X$. If we suppose that there exists $y^{*} \in X$ and $n_{0} \in \mathbb{N}^{*}$ such that $f^{n_{0}}\left(y^{*}\right)=y^{*}$, then the sequence $\left(f^{n}\left(y^{*}\right)\right)_{n \in \mathbb{N}}$ has the constant subsequence $\left(y^{*}\right)_{i_{n_{0}} \in \mathbb{N}}$. It follows that $y^{*}=x^{*}$. So, $F_{f}=F_{f^{n}}=\left\{x^{*}\right\}$, for all $n \in \mathbb{N}^{*}$.

For other set-theoretical results in the fixed point theory see: [27], [28], [29], [48], [46], [56], [65], [79], [154], [102], [103], [104], [108], [117], [155], [6], [99], [156], [146], [77], .. .

For set-theoretical aspects of fixed point theory of multivalued operators see [125] and the references therein.

The above considerations give rise to the following open problems.

Problem I. By P. Urysohn, an $L$-space $(X, \rightarrow)$ is an $L^{*}$-space if the following implication holds:

$\left(x_{n}\right)_{n \in \mathbb{N}} \subset X, x_{n} \not \rightarrow x \Rightarrow$ there exists a subsequence $\left(x_{n_{i}}\right)_{i \in \mathbb{N}}$ of $\left(x_{n}\right)_{n \in \mathbb{N}}$ such that for any subsequence $\left(z_{n}\right)_{n \in \mathbb{N}}$ of $\left(x_{n_{i}}\right)_{i \in \mathbb{N}}$ we have that $z_{n} \not \rightarrow x$. 
We have seen that each $R$-convergence on $X$ is an $L$-convergence on $X$. The problem is which type of $R$-convergence on $X$ is an $L^{*}$-convergence?

References: [43], [21] and the references therein.

Problem II. Conversions between $R$-spaces and metric spaces, and between classes of operators on $R$-spaces to classes of operators on metric spaces.

Commentaries:

Let $(X, R)$ be an $R$-space and $f: X \rightarrow X$ be an operator. Then by definition:

- $f$ is continuous iff,

$\left(x_{n}\right)_{n \in \mathbb{N}} \subset X, x \in X,\left(x_{n}, x\right) \in R_{n}$, for all $n \in \mathbb{N} \Rightarrow\left(f\left(x_{n}\right), f(x)\right) \in R_{n}$, for all $n \in \mathbb{N}$;

- $f$ is $R$-Kannan iff,

$x, y \in X,(x, f(x)) \in R_{n},(y, f(y)) \in R_{n}$, for all $n \in \mathbb{N} \Rightarrow(f(x), f(y)) \in R_{n}$, for all $n \in \mathbb{N}$.

On the other hand, in [66], J. Jachymski constructs on an $R$-space a metric. Moreover, the following result is given.

Jachymski Theorem. Let $X$ be a nonempty set, $f: X \rightarrow X$ be an operator and $l \in] 0,1[$. The following statements are equivalent:

(i) There exists an $R$-structure on $X$ such as in Eilenberg's Theorem.

(ii) There exists a non-Archimedean bounded and complete metric $d$ on $X$ such that $f$ is an l-contraction.

Let $(X, d)$ be a bounded metric space, $f: X \rightarrow X$ be an operator and $l \in] 0,1[$. For $n \in \mathbb{N}$, let $R_{n}:=\left\{(x, y) \mid d(x, y) \leq l^{n} \delta(X)\right\}$. Then $(X, R)$ is an $R$-space. If $f$ is an $R$-contraction, then $f$ satisfies the following metric condition:

$$
x, y \in X, d(x, y) \leq l^{n} \delta(X) \Rightarrow d(f(x), f(y)) \leq l^{n+1} \delta(X) \text {, for all } n \in \mathbb{N} .
$$

The problem is to study the conversions between fixed point theorems in $R$-spaces to fixed point theorems in generalized metric spaces.

Problem III. Structured sets with an $R$-space structure.

Let $(X,+)$ be an abelian group. Let $R=\left(R_{n}\right)_{n \in \mathbb{N}}$ be a complete $R$-space structure on $X$. In addition we suppose that:

$\left(C_{4}\right) z \in X,(x, y) \in R_{n} \Rightarrow(x+z, y+z) \in R_{n}$, for all $n \in \mathbb{N}$.

We call $(X,+, R)$, with $(X,+)$ and $(X, R)$ as above, an $R$-group.

We have the following result (see [116]).

Theorem 2.3. Let $(X,+, R)$ be an $R$-group, with a complete $R$-structure satisfying $\left(C_{4}\right)$ and let $f: X \rightarrow X$ be an $R$-contraction. Then we have that:

(i) $1_{X}-f: X \rightarrow X$ is a bijective operator;

(ii) for each $y \in X$, the operator $g_{y}: X \rightarrow X$ defined by $x \mapsto f(x)+y$, has a unique fixed point $x_{y}^{*}$;

(iii) $\left(g_{y}^{n}(x), x_{y}^{*}\right) \in R_{n}$, for all $n \in \mathbb{N}$.

Proof. From $\left(C_{4}\right)$ we remark that $g_{y}$ is an $R$-contraction, for each $y \in X$. From Theorem 2.1 we have $(i i)$ and $(i i i)$. To finish the proof we observe that $(i i) \Rightarrow(i)$.

The problem is to study the properties of operators on structured sets with $R$-space structure.

\section{EQUiVALENT STATEMENTS WITH THE SET-THEORETICAL ONE}

In the paper [119] we have presented the following deep generalization of a well known converse to the contraction principle due to C. Bessaga (1959, [121], [140]).

Theorem 3.4. Let $X$ be a nonempty set and $f: X \rightarrow X$ be an operator. The following statements are equivalent: 
(1) $F_{f}=F_{f^{n}} \neq \emptyset$, for all $n \in \mathbb{N}^{*}$.

(2) For each $l \in] 0,1[$ there exists a complete metric $d$ on $X$ such that:

(a) $f:(X, d) \rightarrow(X, d)$ is orbitally continuous;

(b) $d\left(f^{2}(x), f(x)\right) \leq l d(x, f(x))$, for all $x \in X$, i.e., $f$ is an l-graphic contraction;

(3) There exists a complete metric $d$ on $X$ such that $f:(X, d) \rightarrow(X, d)$ is a weakly Picard operator.

Theorem 1 in [119] contains three other equivalent statements.

Revisiting this result and others that are similar, in what follows we give the following result.

Theorem 3.5 (Theorem of equivalent statements). Let $X$ be a nonempty set and $f: X \rightarrow X$ be an operator. The following statements are equivalent:

(1) $F_{f}=F_{f^{n}} \neq \emptyset$, for all $n \in \mathbb{N}^{*}$.

(2) There exists an $L$-space structure on $X$ with respect to which $f$ is weakly Picard operator.

(3) For each $l \in] 0,1[$ there exists a complete metric $d$ on $X$ such that:

(a) $f$ is an l-graphic contraction with respect to the metric $d$;

(b) $f$ is orbitally continuous with respect to the metric $d$.

(4) $F_{f} \neq \emptyset$ and there exists a metric $d$ on $X$ with respect to which $f$ is asymptotically regular.

(5) There exists a metric $d$ on $X$ and a partition of $X, X=\bigcup_{\lambda \in \Lambda} X_{\lambda}$, such that:

(a) $f\left(X_{\lambda}\right) \subset X_{\lambda}$, for all $\lambda \in \Lambda$;

(b) $\left.f\right|_{X_{\lambda}}: X_{\lambda} \rightarrow X_{\lambda}$ is a Picard operator with respect to the metric d, for all $\lambda \in \Lambda$.

Proof. For $(1) \Leftrightarrow(3)$ and $(3) \Leftrightarrow(5)$ see [119].

$(2) \Rightarrow(1)$. Since $f$ is a weakly Picard operator in $(X, \rightarrow)$, it follows that,

$$
f^{n}(x) \rightarrow f^{\infty}(x) \text { as } n \rightarrow \infty \text {. }
$$

Let us suppose that there exists $n_{0} \in \mathbb{N}^{*}$ and $y \in X$ such that $f^{n_{0}}(y)=y$. The sequence $\left(f^{n}(y)\right)_{n \in \mathbb{N}}$ converges to the fixed point of $f, f^{\infty}(y)$. But the constant sequence $(y)$ is a subsequence of $\left(f^{n}(y)\right)_{n \in \mathbb{N}}$. So, $y=f(y)$.

$(1) \Rightarrow(2)$. Follows from $(1) \Rightarrow(3) \Rightarrow(2)$.

$(4) \Rightarrow(1)$. For some $n_{0} \in \mathbb{N}^{*}$, let $y \in F_{f^{n_{0}}}$. Since $f$ is asymptotically regular, the sequence, $\left(d\left(f^{k n_{0}}(y), f^{k n_{0}+1}(y)\right)_{k \in \mathbb{N}}=(d(y, f(y)))_{k \in \mathbb{N}} \rightarrow 0\right.$ as $k \rightarrow \infty$. So, $y=f(y)$.

$(1) \Rightarrow(4)$. Follows from $(1) \Rightarrow(3) \Rightarrow(4)$.

For other statements equivalent with $F_{f}=F_{f^{n}} \neq \emptyset, n \in \mathbb{N}^{*}$, see J. Jachymski [65]. See also Rus-Petruşel-Şerban [141], V.G. Angelov [8].

Here are some references for the terms which appear in the Equivalent Statements Theorem:

- Weakly Picard operators: [122], [121], [140], [135], [131], [21], [143], [134], [22], [29], $\cdots$

- graphic contractions: [105], [106], [140], [143], [92], ...

- asymptotic regular operators: [137], [17], [21], [23] and the references therein.

Problem IV. To give equivalent statements with the set-theoretical statement:

$$
F_{f}=F_{f^{n}} \neq \emptyset \text {, for all } n \in \mathbb{N}^{*} .
$$

Problem V. To give equivalent statements with the set-theoretical statement:

$$
F_{f}=F_{f^{n}}=\left\{x^{*}\right\} .
$$

Problem VI. To give equivalent statements with the set-theoretical statement:

$$
\bigcap_{n \in \mathbb{N}} f^{n}(X)=\left\{x^{*}\right\} .
$$


Problem VII. To give equivalent statements with the set-theoretical statement:

$$
\bigcap_{n \in \mathbb{N}} f^{n}(X)=F_{f}
$$

Commentaries:

Let $X$ be a nonempty set and $f: X \rightarrow X$ be an operator. If $\bigcap_{n \in \mathbb{N}} f^{n}(X)=\left\{x^{*}\right\}$, then $F_{f}=F_{f^{n}}=\left\{x^{*}\right\}$.

We have the following results.

Theorem 3.6. Let $X$ be a nonempty set and $f: X \rightarrow X$ be an operator. The following statements are equivalent:

(1) $F_{f}=F_{f^{n}}=\left\{x^{*}\right\}$.

(2) For each $l \in] 0,1[$ there exists a complete metric on $X$ such that $f:(X, d) \rightarrow(X, d)$ is an l-contraction.

(3) There exist $x^{*} \in X$ and a metric $d$ on $X$ such that:

(a) $F_{f}=\left\{x^{*}\right\}$;

(b) $f:(X, d) \rightarrow(X, d)$ is asymptotically regular.

(4) There exists an L-space structure on $X$ such that $f:(X, \rightarrow) \rightarrow(X, \rightarrow)$ is a Picard operator.

Proof. $(1) \Leftrightarrow(2)$. This is Bessaga's Theorem.

$(3) \Rightarrow(1)$. From $(b)$ we have that $F_{f}=F_{f^{n}}$, for all $n \in \mathbb{N}^{*}$.

$(1) \Rightarrow(3)$. Follows from $(1) \Rightarrow(2) \Rightarrow(3)$.

$(4) \Rightarrow(1)$. Follows from the definition of a Picard operator.

$(1) \Rightarrow(4)$. Follows from $(1) \Rightarrow(2)$ and the fact that $(X, \stackrel{d}{\rightarrow})$ is an $L$-space.

It is well-known the following result.

Janos Theorem. Let $(X, d)$ be a compact metric space and $f: X \rightarrow X$ be such that:

(i) $f$ is continuous;

(ii) $\bigcap_{n \in \mathbb{N}} f^{n}(X)=\left\{x^{*}\right\}$.

Then for each $l \in] 0,1[$ there exists a metric $\rho$ on $X$ such that:

(a) $d$ and $\rho$ are topologically equivalent;

(b) $f:(X, \rho) \rightarrow(X, \rho)$ is an l-contraction.

For the following set-theoretical notions: Bessaga operators (i.e., $F_{f}=F_{f^{n}}=\left\{x^{*}\right\}$, for all $n \in \mathbb{N}$ ), Rus operators (i.e., $F_{f}=F_{f^{n}} \neq \emptyset$, for all $n \in \mathbb{N}^{*}$ ) and Janos operators (i.e., $\left.\bigcap_{n \in \mathbb{N}} f^{n}(X)=\left\{x^{*}\right\}\right)$ see: [109], [121], [140], [131], [143], [85], [138], [83], ..

\section{INVARIANT OPERATOR WITH RESPECT TO AN OPERATOR: INVARIANT PARTITION OF A SET WITH RESPECT TO AN OPERATOR}

One of the basic problem in the weakly Picard operator theory is the following.

Problem VIII. Let $(X, \rightarrow)$ be an $L$-space ( $R$-space, topological space, metric space, Banach space, Hilbert space, ordered $L$-space, ...) and $f: X \rightarrow X$ be an operator. The problem is to give conditions on $X$ and $f$ which imply that $f$ is a weakly Picard operator.

In which terms shall we give these conditions?

It is natural to start with set-theoretical terms. With the Theorem of Equivalent Statements (Theorem 3.5) in mind, one of them is the following. 
Definition 4.4. Let $X$ be a nonempty set and $f: X \rightarrow X$ be an operator. By definition, a partition of $X=\bigcup_{\lambda \in \Lambda} X_{\lambda}$ is an invariant partition with respect to the operator $f$ if $f\left(X_{\lambda}\right) \subset$ $X_{\lambda}$, for all $\lambda \in \Lambda$. If $F_{f} \neq \emptyset, \Lambda=F_{f}$ and $F_{f} \cap X_{x^{*}}=\left\{x^{*}\right\}$, for all $x^{*} \in F_{f}$, then the corresponding invariant partition is called fixed point invariant partition of $X$.

In order to generate invariant partitions, the following terms are useful.

Definition 4.5. [[38], p. 299, [133], [33], [96]] Let $X$ and $Y$ be two sets and $f: X \rightarrow X$ be an operator. By definition, an operator $\Phi: X \rightarrow Y$ is invariant with respect to $f$ (or is invariant operator for $f$ ) iff $\Phi \circ f=\Phi$.

It is important to remark that each surjective invariant operator $\Phi: X \rightarrow Y$ of an operator $f: X \rightarrow X$ generates an invariant partition of $X$ with respect to the operator $f$, as follows. For $y \in Y$, let $X_{y}:=\Phi^{-1}(y)$. Then $X=\bigcup_{y \in Y} X_{y}$ is an invariant partition of $X$ with respect to the operator $f$.

For a better understanding of the above notions it is useful to see the following examples.

Example 4.1. [Interpolation set of an operator and invariant partition]

Let $X, Y$ be nonempty sets, $\mathbb{M}(X, Y):=\{f: X \rightarrow Y \mid f$ is an operator $\}$ and $T$ : $\mathbb{M}(X, Y) \rightarrow \mathbb{M}(X, Y)$ be an operator. By definition, a subset $I \subset X$ is an interpolation set for $T$ iff

$$
T(f)(x)=f(x), \text { for all } f \in \mathbb{M}(X, Y) \text { and all } x \in I .
$$

Let $\Phi: \mathbb{M}(X, Y) \rightarrow \mathbb{M}(I, Y)$ be defined by $\left.f \mapsto f\right|_{I}$, where $\left.f\right|_{I}$ is the restriction of $f$ to $I$. It is clear that $\Phi \circ T=\Phi$, i.e. $\Phi$ is an invariant operator of $T$. So, $\Phi$ generates the following invariant partition of the set $Z:=\mathbb{M}(X, Y)$,

$$
Z=\bigcup_{\lambda \in \mathbb{M}(I, Y)} Z_{\lambda}, \text { where } Z_{\lambda}:=\left\{f \in \mathbb{M}(X, Y)|f|_{I}=\lambda\right\} .
$$

Example 4.2. Let $(X, \rightarrow)$ be an $L$-space and $f: X \rightarrow X$ be a weakly Picard operator. Then, the operator $\Phi:=f^{\infty}: X \rightarrow F_{f}$ is an invariant operator of $f$.

Example 4.3. Let $B$ be a Banach space and $T: C([a, b], B) \rightarrow C([a, b], B)$ be defined by

$$
T(f)(x):=f(a)+\int_{a}^{x} K(x, s, f(s)) d s, x \in[a, b],
$$

where $K \in C([a, b] \times[a, b] \times B, B)$. Then $a$ is an interpolation point of the operator $T$ and $\Phi: C([a, b], B) \rightarrow B$, defined by $\Phi(f):=f(a)$, is an invariant operator for $T$. For $\lambda \in B$, let $Z_{\lambda}:=\{f \in C([a, b], B) \mid f(a)=\lambda\}$. Then $C([a, b], B)=\bigcup_{\lambda \in B} Z_{\lambda}$ is an invariant partition with respect to the operator $T$. If $K(t, s, \cdot): B \rightarrow B$ is $L$-Lipschitz for $t, s \in[a, b]$, then $\left.T\right|_{Z_{\lambda}}: Z_{\lambda} \rightarrow Z_{\lambda}$ is a contraction with respect to a suitable Bielecki norm on $Z_{\lambda}$. From these we have that the operator $T$ is a weakly Picard operator with respect to the uniform convergence on $C([a, b], B)$.

Example 4.4. Let $B$ be a Banach space, $h>0$ and $T: C([a-h, b], B) \rightarrow C([a-h, b], B)$, be defined by

$$
T(f)(x):= \begin{cases}f(x), & x \in[a-h, a] \\ f(a)+\int_{a}^{x} K(x, s, f(s), f(s-h)) d s, & x \in[a, b]\end{cases}
$$


where $K \in C([a, b] \times[a, b] \times B \times B, B)$. Then $I:=[a-h, a]$ is an interpolation set for $T$ and $\Phi: C([a-h, b], \mathbb{R}) \rightarrow C([a-h, a], B),\left.f \mapsto f\right|_{[a-h, b]}$ is an invariant operator of $T$. For $\lambda \in C([a-h, b], B)$, let $Z_{\lambda}:=\left\{f \in C([a-h, b], B)|f|_{[a-h, a]}=\lambda\right\}$. Then $C([a-h, b], B)=\bigcup_{\lambda \in C([a-h, a], B)} Z_{\lambda}$ is an invariant partition with respect to the operator $T$. If $K(t, s, \cdot, \cdot): B \times B \rightarrow B$ is $L$-Lipschitz for all $t, s \in[a, b]$ then $\left.T\right|_{Z_{\lambda}}: Z_{\lambda} \rightarrow Z_{\lambda}$ is a contraction with respect to a suitable Bielecki norm on $Z_{\lambda}$. These imply that the operator $T$ is a weakly Picard operator with respect to the uniform convergence on $C([a-h, b], B)$.

Example 4.5. Let $T: C[0,1] \rightarrow C[0,1]$ be a linear positive operator such that:

$$
F_{T}=\left\{f \in C[0,1] \mid f(x)=c_{1} x+c_{2}, c_{1}, c_{2} \in \mathbb{R}\right\} .
$$

Then it is well known that (see Rus [127] and the references therein) the set $I=\{0,1\}$ is an interpolation set for $T$. Then $\Phi: C[0,1] \rightarrow \mathbb{R}^{2}$, defined by $\Phi(f):=(f(0), f(1))$, is an invariant operator of $T$. For $\lambda \in \mathbb{R}^{2}$, let $Z_{\lambda}:=\left\{f \in C[0,1] \mid f(0)=\lambda_{1}, f(1)=\lambda_{2}\right\}$. Then $C[a, b]=\bigcup_{\lambda \in \mathbb{R}^{2}} Z_{\lambda}$ is an invariant partition with respect to $T$. If, for example, $T=B_{n}$, is a Bernstein operator, $n \in \mathbb{N}^{*}$ (see Rus [123]),

$$
B_{n}(f)(x):=\sum_{k=0}^{n} f\left(\frac{k}{n}\right)\left(\begin{array}{l}
n \\
k
\end{array}\right) x^{k}(1-x)^{n-k}, x \in[0,1]
$$

then we are in the above conditions, and $B_{n}: Z_{\lambda} \rightarrow Z_{\lambda}$, is a contraction with respect to the max-norm on $C[0,1]$. So, the Bernstein operators are weakly Picard operators with respect to the uniform convergence on $C[0,1]$.

Example 4.6. [Petruşel-Rus-Şerban [95]] Let $f: \mathbb{R}^{m} \rightarrow \mathbb{R}^{m}$ be a linear positive stochastic operator. Then the functional $\Phi: \mathbb{R}^{m} \rightarrow \mathbb{R}$, defined by $\Phi\left(x_{1}, \ldots, x_{m}\right):=\sum_{k=1}^{m} x_{k}$ is an invariant functional of $f$. For $\lambda \in \mathbb{R}$, let $X_{\lambda}:=\left\{x \in \mathbb{R}^{m} \mid \sum_{k=1}^{m} x_{k}=\lambda\right\}$. Then $\mathbb{R}^{m}=\bigcup_{\lambda \in \mathbb{R}} X_{\lambda}$ is an invariant partition with respect to $f$ and $\left.f\right|_{X_{\lambda}}: X_{\lambda} \rightarrow X_{\lambda}$ is a contraction with respect to the $\|\cdot\|_{1}$ on $\mathbb{R}^{m}$, for all $\lambda \in \mathbb{R}$. It follows that $f$ is a weakly Picard operator.

Example 4.7. [András-Rus [5]] Let $(\mathbb{B},\|\cdot\|)$ be a Banach space. For $x \in \mathbb{R}, \tilde{x}:=(x, \ldots, x, \ldots)$ is the constant sequence defined by $x$. We denote by $s(\mathbb{B})$ the set of all sequences with elements in $\mathbb{B}$. We consider the $L$-space, $(s(\mathbb{B}), \stackrel{t}{\rightarrow})$, where $\stackrel{t}{\rightarrow}$ is the termwise convergence on $s(\mathbb{B})$. Also, we consider on $s(\mathbb{B})$ the family of pseudometrics, $\mathscr{D}:=\left\{d_{k} \mid k \in\right.$ $\mathbb{N}$, where $\left.d_{k}(u, v):=\max _{0 \leq n \leq k}\left\|u_{n}-v_{n}\right\|\right\}$. Then the gauge space $(s(\mathbb{B}), \mathscr{D})$ is separated and complete. Moreover for $\left(u^{n}\right)_{n \in \mathbb{N}}, u \in s(\mathbb{B})$, we have that

$$
u^{n} \stackrel{\mathscr{D}}{\rightarrow} \text { u as } n \rightarrow \infty \Rightarrow u^{n} \stackrel{t}{\rightarrow} \text { u as } n \rightarrow \infty .
$$

Let us consider the Cesáro operator, $C: s(\mathbb{B}) \rightarrow s(\mathbb{B})$. This operator is defined by

$$
\left(u_{0}, u_{1}, \ldots, u_{n}, \ldots\right) \mapsto\left(u_{0}, \frac{1}{2}\left(u_{0}+u_{1}\right), \ldots, \frac{1}{n+1}\left(u_{0}+u_{1}+\ldots+u_{n}\right), \ldots\right) .
$$

We remark that the fixed point set of $C$ is $F_{C}=\{\tilde{x} \mid x \in \mathbb{B}\}$.

For $x \in \mathbb{B}$ we consider $X_{x}:=\left\{u \in s(\mathbb{B}) \mid u_{0}=x\right\}$. Then $s(\mathbb{B})=\bigcup_{x \in \mathbb{B}} X_{x}$ is an invariant partition with respect to $C$. We remark that $X_{x}$ is a closed subset of $s(\mathbb{B})$ and $\left.C\right|_{X_{x}}: X_{x} \rightarrow$ $X_{x}$ is a contraction with respect to the family of pseudometrics $\mathscr{D}$. 
So, the operator $C:(s(\mathbb{B}), \stackrel{t}{\rightarrow}) \rightarrow(s(\mathbb{B}), \stackrel{t}{\rightarrow})$ is a weakly Picard operator.

A particular case of the Problem VIII is the following.

Problem IX (Rus, [131]). Let $(X, \rightarrow)$ be an $L$-space, $f: X \rightarrow X$ be an operator with $F_{f} \neq \emptyset$. The problem is in which conditions there exists an invariant subset $Y$ of $f$ such that:

$$
\left.f\right|_{Y}: Y \rightarrow Y \text { is } W P O \Rightarrow f: X \rightarrow X \text { is } W P O \text { ? }
$$

Regarding the technique of invariant partitions in studying the Probelms VIII and IX see: Rus ([123], [133], [122]), Agratini-Rus [3], J. Jachymski [67], András-Rus [5], GonskaKacsó-Piţul [52], Gonska-Piţul [53], Cătinaş-Otrocol-Rus [33], Petruşel-Rus-Şerban ([96], [95]), Bacoţiu [12], Cătinaş-Otrocol [32], ...

Regarding the interpolation set see [127] and the references therein (Altomare-Campiti (1994), Boboc-Bucur (1976), Gavrea-Ivan (2005), Raşa (2009)).

For some applications to differential and integral equations see Rus [128] and the references therein (Bacoţiu (2008), Buică-Ilea (2007), Dincuţă (2000), Dobriţoiu (2009), E. Egri (2008), R. Gabor (2006), V. Mureşan (2003), Olaru (2010), Otrocol (2005), Şerban (2002)).

\section{FiXED POINT STRUCTURES ON AN ABSTRACT SET}

Another way to use set-theoretical terms in the fixed point theory started to be constructed in 1986 ([111], [112], [113], [118], ...). In this section we revisit this construction.

5.1. Examples of fixed point structures. For two sets $X, Y$, we denote by

$$
\mathbb{M}(X, Y):=\{f: X \rightarrow Y \mid f \text { is an operator }\}
$$

and by

$$
\mathbb{M}(X):=\mathbb{M}(X, X) .
$$

Let $M$ be an operator defined as follows

$$
M: D_{M} \subset P(X) \times P(X) \multimap \bigcup_{U, V \in P(X)} \mathbb{M}(U, V),
$$

such that $(U, V) \mapsto M(U, V) \subset \mathbb{M}(U, V)$.

We denote by $M(U):=\mathbb{M}(U, U)$.

Definition 5.6. By a fixed point structure (f.p.s.) on a nonempty set $X$ we understand a triple $(X, S(X), M)$ with the following properties:

(i) $S(X) \subset P(X)$ and $U \in S(X) \Rightarrow(U, U) \in D_{M}$;

(ii) $U \in S(X), f \in M(U) \Rightarrow F_{f} \neq \emptyset$;

(iii) $M$ is such that

$$
(Y, Y) \in D_{M}, Z \in P(Y),(Z, Z) \in D_{M} \Rightarrow M(Z) \supset\left\{\left.f\right|_{Z} \mid f \in M(Y)\right\} .
$$

A triple $(X, S(X), M)$ which satisfies $(i)$ and $(i i)$ is called a large fixed point structure (l.f.p.s.).

Example 5.8. [The trivial f.p.s.] $X$ is a set, $S(X):=\{\{x\} \mid x \in X\}$ and for $Y \in P(X)$, $M(Y):=\mathbb{M}(Y)$.

Example 5.9. [The f.p.s. of $R$-contractions] $(X, R)$ is a complete $R$-space, $S(X)=\{X\}$ and $M(X):=\{f: X \rightarrow X \mid f$ is an $R$ - contraction $\}$.

Example 5.10. [The f.p.s. of contractions] $(X, d)$ is a complete metric space, $S(X):=$ $P_{c l}(X):=\{Y \in P(X) \mid Y=\bar{Y}\}$ and $M(Y):=\{f: Y \rightarrow Y \mid f$ is a contraction $\}$.

Example 5.11. [the f.p.s of Tarski] $(X, \leq)$ is a complete lattice, $S(X):=\{X\}$ and $M(Y):=$ $\{f: Y \rightarrow Y \mid f$ is increasing $\}$. 
Example 5.12. [The f.p.s. of progressive operators] $(X, \leq)$ is a partially ordered set, $S(X):=\{Y \in P(X) \mid(Y, \leq)$ has a maximal element $\}$ and $M(Y):=\{f: Y \rightarrow Y \mid x \leq$ $f(x)$, for all $x \in Y\}$.

Example 5.13. [The f.p.s. of Schauder] $X$ is a Banach space, $S(X):=P_{c p, c v}(X)$ and $M(Y):=C(Y, Y)$.

Example 5.14. [The f.p.s. of Browder-Ghöde-Kirk] $X$ is a uniformly convex Banach space, $S(X):=P_{b, c l, c v}(X)$ and $M(Y):=\{f: Y \rightarrow Y \mid f$ is nonexpansive $\}$.

Example 5.15. [The f.p.s. of Nemytzki-Edelstein] $(X, d)$ is a metric space, $S(X):=P_{c p}(X)$ and $M(Y):=\{f: Y \rightarrow Y \mid f$ is a contractive operator $\}$.

It is clear that for each fixed point theorem we have at least a f.p.s.

Here are the basic problems of the f.p.s. theory.

Problem X (Maximal f.p.s.) Which are the f.p.s. $(X, S(X), M)$ with the following property: there exists $S_{1}(X) \supset S(X)$ such that $S(X)=\left\{Y \in S_{1}(X) \mid f \in M(Y) \Rightarrow F_{f} \neq \emptyset\right\}$ ?

Problem XI (f.p.s. with the common fixed point property). Which are the f.p.s. $(X, S(X), M)$ with the following property:

$$
Y \in S(X), f, g \in M(Y), f \circ g=g \circ f \Rightarrow F_{f} \cap F_{g} \neq \emptyset ?
$$

Problem XII (f.p.s. with the coincidence property). Which are the f.p.s. $(X, S(X), M)$ with the following property:

$$
Y \in S(X), f, g \in M(Y), f \circ g=g \circ f \Rightarrow\{x \in Y \mid f(x)=g(x)\} \neq \emptyset ?
$$

References: [126], [130], [111], [112], [113], [117], [118], [140], [96], [136], [149], [153], [10], [139].

5.2. Compatible pair with a fixed point structure. Let $(X, S(X), M)$ be a f.p.s., $\theta: Z \rightarrow$ $\mathbb{R}_{+}$, with $S(X) \subset Z \subset P(X)$ and $\eta: \mathcal{P}(X) \rightarrow \mathcal{P}(X)$. By definition ([126], p. 52), the pair $(\theta, \eta)$ is a compatible pair with $(X, S(X), M)$ iff:

(1) $\eta$ is a closure operator, $S(X) \subset \eta(Z) \subset Z$ and $\theta(\eta(Y))=\theta(Y)$, for all $Y \in Z$;

(2) $F_{\eta} \cap Z_{\theta} \subset S(X)$, where $Z_{\theta}:=\{A \in Z \mid \theta(A)=0\}$.

Example 5.16. On a metric space $(X, d)$ let $(X, S(X), M)$ be the f.p.s. of Nemytski-Edelstein. Let $Z:=P_{b}(X), \theta=\alpha_{k}$ - the Kuratowski measure of noncompactness on $X$ and $\eta(Y)=\bar{Y}$. Then $(\theta, \eta)$ is a compatible pair with $(X, S(X), M)$.

Example 5.17. On a Banach space $X$, let $(X, S(X), M)$ be the f.p.s. of Schauder. Let $Z:=P_{b}(X), \theta:=\alpha_{k}$ and $\eta(Y)=\overline{c o} Y$ - the closed convex hull of $Y$. Then $(\theta, \eta)$ is a compatible pair with the fixed point structure of Schauder on $X$.

Another problem concerning the f.p.s. theory is the following.

Problem XIII. Let $(X, S(X), M)$ be a f.p.s. on a structured set $X$. The problem is to look at the compatible pair with $(X, S(X), M)$.

References: Rus [126] and [130], and the references therein.

5.3. Closure operators and invariant subsets. Let $X$ be a nonempty set. By definition, an operator $\eta: \mathcal{P}(X) \rightarrow \mathcal{P}(X)$ is a closure operator iff:

(i) $Y \subset \eta(Y)$, for all $Y \subset X$;

(ii) $Y, Z \subset X, Y \subset Z \Rightarrow \eta(Y) \subset \eta(Z)$;

(iii) $\eta \circ \eta=\eta$. 
Here are some examples of closure operators.

In a real linear space $X$, a convex hull operator, $\eta: \mathcal{P}(X) \rightarrow \mathcal{P}(X), Y \mapsto c o Y$, is a closure operator.

In a topological space $X$, the operator $\eta: \mathcal{P}(X) \rightarrow \mathcal{P}(X), Y \mapsto \bar{Y}$ is a closure operator.

In a linear topological space, the operator, $\eta: \mathcal{P}(X) \rightarrow \mathcal{P}(X), Y \mapsto \overline{c o} Y:=\overline{c o Y}$, is a closure operator.

Another example is the following (see Rus-Şerban [144]).

Let $X_{i}, i=\overline{1, m}$ be some nonempty sets and $X:=X_{1} \times X_{2} \times \ldots \times X_{m}$ be their cartesian product. Let us denote by, $\Pi_{i}: X \rightarrow X_{i}$ the canonical projection of $X$ on $X_{i}, i=\overline{1, m}$. If $Y \subset X$, then the cartesian hull of $Y$ is

$$
c a Y:=\Pi_{1}(Y) \times \ldots \Pi_{m}(Y) .
$$

The operator $c a: \mathcal{P}(X) \rightarrow \mathcal{P}(X)$, defined by $Y \mapsto c a Y$ is a closure operator.

Other examples of closure operators come from generalized notions of convexity. See for example: I. Singer [152], R. Precup [98] and I.A. Rus [132].

In the terms of closure operators we have the following general result.

General Invariant Subset Lemma (Rus [126], p. 21). Let $X$ be a nonempty set, $\eta: \mathcal{P}(X) \rightarrow$ $\mathcal{P}(X)$ be a closure operator, $Y \in F_{\eta}$ be a set, $y \in Y$ be a point and $f: Y \rightarrow Y$ be an operator. Then there exists a subset $Y_{0} \subset Y$ such that:

(1) $y \in Y_{0}$;

(2) $Y_{0} \in F_{\eta}$;

(3) $f\left(Y_{0}\right) \subset Y_{0}$;

(4) $\eta\left(f\left(Y_{0}\right) \cup\{y\}\right)=Y_{0}$.

5.4. Fixed point theorems in terms of a compatible pair: $\theta$-condensing operators. Let $X$ be a nonempty set, $Z \subset P(X), Z \neq \emptyset$ and $\theta: Z \rightarrow \mathbb{R}_{+}$be a functional. An operator $f: X \rightarrow X$ is strongly $\theta$-condensing iff:

(1) $A \in Z \Rightarrow f(A) \in Z$;

(2) $A \in Z, \theta(A) \neq 0 \Rightarrow \theta(f(A))<\theta(A)$.

If in the above definition, instead of $(2)$ we have,

$\left(2^{\prime}\right) A \in Z, f(A) \subset A, \theta(A) \neq 0 \Rightarrow \theta(f(A))<\theta(A)$,

then the operator $f$ is called $\theta$-condensing.

One of the basic set-theoretical fixed point result, in terms of a fixed point structure is the following.

Theorem 5.7. Let $(X, S(X), M)$ be a f.p.s., $(\theta, \eta)\left(\theta: Z \rightarrow \mathbb{R}_{+}\right)$be a compatible pair with $(X, S(X), M)$. Let $Y \in \eta(Z)$ and $f \in M(Y)$. We suppose that:

(i) $A \in Z, x \in Y$ imply that $A \cup\{x\} \in Z$ and $\theta(A \cup\{x\})=\theta(A)$;

(ii) $f$ is a $\theta$-condensing operator.

Then we have that:

(a) there exists $A_{0} \in S(X)$ such that $f\left(A_{0}\right) \subset A_{0}$;

(b) $F_{f} \neq \emptyset$;

(c) if $F_{f} \in Z$, then $\theta\left(F_{f}\right)=0$.

Proof. $(a)+(b)$. For $x \in Y$, by the General Invariant Subset Lemma, there exists $A_{0} \in F_{f}$ with $f\left(A_{0}\right) \subset A_{0}$ and $\eta\left(f\left(A_{0}\right) \cup\{x\}\right)=A_{0}$. We have

$$
\theta\left(\eta\left(f\left(A_{0}\right) \cup\{x\}\right)\right)=\theta\left(f\left(A_{0}\right) \cup\{x\}\right)=\theta\left(f\left(A_{0}\right)\right)=\theta\left(A_{0}\right)
$$


Since $f$ is $\theta$-condensing it follows that $\theta\left(A_{0}\right)=0$, i.e., $A_{0} \in Z_{\theta}$. From $A_{0} \in F_{\eta} \cap Z_{\theta}$, $f\left(A_{0}\right) \subset A_{0}$ and $\left.f\right|_{A_{0}} \in M\left(A_{0}\right)$, and $(\theta, \eta)$ is a compatible pair with $(X, S(X), M)$ we have that $F_{f} \neq \emptyset$.

$(c)$. By $F_{f} \in Z$ and $f\left(F_{f}\right)=F_{f}$ it follows that $\theta\left(F_{f}\right)=0$.

From the proof of Theorem 5.7 we have:

Theorem 5.8. In the Theorem 5.7 instead of condition (ii) we can put the following one:

$\left(i i^{\prime}\right) f: f(Y) \rightarrow f(Y)$ is $\theta$-condensing.

Our Theorems 5.7 and 5.8 are set-theoretical generalizations of many known fixed point theorems of Sadowski type (B.N. Sadowski (1967), H. Amann (1973), J. Appell (2005), J. Banas and K. Goebel (1980), S. Czerwik (1980), G. Emmanuele (1981), M. Furi - A. Vignoli (1969), J.K. Hale - O. López (1973), K. Iseki (1976), ...).

For example, if $X$ is a Banach space and $(X, S(X), M)$ is a Schauder f.p.s., $Z:=P_{b}(X)$, $\eta:=\overline{c o}$ and $\theta:=\alpha_{H}$ - the Hausdorff measure of noncompactness and $f$ is a strong $\alpha_{H}$ - condensing operator, from Theorem 5.7, we have the well known Sadowski fixed point theorem.

\section{CLOSURE OPERATORS AND INVARIANT SUBSET IN TERMS OF ITERATES: $(\theta, l)$-CONTRACTIONS}

6.1. Invariant subsets. Let $X$ be a nonempty set, $\eta: \mathcal{P}(X) \rightarrow \mathcal{P}(X)$ be a closure operator, $Y \in F_{\eta}$ and $f: Y \rightarrow Y$ be an operator. Let

$$
Y_{1}:=\eta(f(Y)), Y_{2}:=\eta\left(f\left(Y_{1}\right)\right), \ldots, Y_{n}:=\eta\left(f\left(Y_{n-1}\right)\right), n \in \mathbb{N}^{*} .
$$

We remark that $Y_{n+1} \subset Y_{n}, Y_{n} \in F_{\eta}$ and $Y_{n} \in I(f):=\{A \subset Y \mid f(A) \subset A\}$. Let us denote $Y_{\infty}:=\bigcap_{n \in \mathbb{N}} Y_{n}$. It is clear that $Y_{\infty}$ is an invariant subset of $f$.

The following problem rises.

Problem XIV. Let $(X, S(X), M)$ be a f.p.s. on a set $X$ and $f \in M(Y)$. The problem is in which conditions we have that: $Y_{\infty} \neq \emptyset$ and $Y_{\infty} \in S(X)$ ?

If $f$ is a solution of Problem XIV, then $F_{f} \neq \emptyset$.

In 1986 we started to study the Problem XIV. Till now, it stills open.

To study the problem we introduced two notions: operator with intersection property and $(\theta, l)$-contraction.

6.2. Operators with intersection property. Let $(O, \rightarrow, \leq)$ be an ordered $L$-space with the least element, 0 . Let $X$ be a nonempty set, $Z \subset P(X), Z \neq \emptyset$. By definition, an operator $\theta: Z \rightarrow O$ has the intersection property if $Y_{n} \in Z, Y_{n+1} \subset Y_{n}, n \in \mathbb{N}$ and $\theta\left(Y_{n}\right) \rightarrow 0$ as $n \rightarrow \infty$, imply that:

$$
Y_{\infty}:=\bigcap_{n \in \mathbb{N}} Y_{n} \neq \emptyset, Y_{\infty} \in Z \text { and } \theta\left(Y_{\infty}\right)=0 .
$$

Example 6.18. Let $(X, d)$ be a complete metric space, $Z:=P_{b, c l}(X), O:=\mathbb{R}_{+}$. Then $\delta, \alpha_{k}$ and $\alpha_{H}$ are functionals with intersection property.

Example 6.19. Let $X$ be a locally convex space and $\left(p_{i}\right)_{i \in I}$ be a family of seminorms which generates the topology on $X$. Let $Z:=P_{b, c l}(X)$ and $O:=\mathbb{M}\left(I, \mathbb{R}_{+}\right)$. We define the operator, $\theta: Z \rightarrow \mathbb{M}\left(I, \mathbb{R}_{+}\right)$, by $\theta(A):=$ with the function $i \mapsto \alpha_{K}^{i}(A)$, where $\alpha_{K}^{i}$ is the Kuratowski measure of noncompactness with respect to the seminorm $p_{i}$. In a similar way we can define $\theta$ with respect to the diameter functional, $\delta$, and to the Hausdorff measure of noncompactness, $\alpha_{H}$. The operator $\theta$ has the intersection property. 
For other examples of such operators see [126], pp. 41-55.

Problem XV. To look for examples of operators with intersection property.

6.3. Fixed point theorems in terms of an operator with intersection property: $(\theta, l)$ contraction. We are starting by introducing the notion of $(\theta, l)$-contraction.

Let $X$ be a nonempty set, $Z \subset P(X), Z \neq \emptyset, \theta: Z \rightarrow \mathbb{R}_{+}$be an operator and $\left.l \in\right] 0,1[$.

Definition 6.7. An operator $f: X \rightarrow X$ is a $(\theta, l)$-contraction iff:

(i) $A \in Z \Rightarrow f(A) \in Z$;

(ii) $\theta(f(A)) \leq l \theta(A)$, for all $A \in Z$ with $f(A) \subset A$.

Definition 6.8. An operator $f: X \rightarrow X$ is a strongly $(\theta, l)$-contraction iff:

(i) $A \in Z \Rightarrow f(A) \in Z$;

(ii) $\theta(f(A)) \leq l \theta(A)$, for all $A \in Z$.

Example 6.20. Let $(X, d)$ be a metric space, $Z:=P_{b}(X)$ and $\theta:=\delta$. Then an operator $f: X \rightarrow X$ is a $(\delta, l)$-contraction iff: $A \in P_{b}(X) \Rightarrow f(A) \in P_{b}(X)$ and $\delta(f(A)) \leq l \delta(A)$, for all $A \in P_{b}(X)$ with $f(A) \subset A$.

It is important to remark that $f$ is a strong $(\delta, l)$-contraction iff $f$ is an $l$-contraction.

Example 6.21. Let $(X, d)$ be a metric space and $Z:=P_{b}(X)$. If $f: X \rightarrow X$ is a compact operator, then $f$ is an $\left(\alpha_{K}, l\right)$-contraction, for each $\left.l \in\right] 0,1[$.

Example 6.22. The radial retraction on a Banach space $X$ to $\bar{B}(0 ; 1)$ is a strong $\alpha_{K}$-nonexpansive mapping, i.e.

$$
\alpha_{K}(\rho(A)) \leq \alpha_{K}(A), \text { for all } A \in P_{b}(X) .
$$

My set-theoretical fixed point results for (strong) $(\theta, l)$-contractions are the following (see [126], pp. 69-70).

Theorem 6.9. Let $(X, S(X), M)$ be a f.p.s. on a set $X,(\theta, \eta)\left(\theta: Z \rightarrow \mathbb{R}_{+}\right)$be a compatible pair with $(X, S(X), M)$. Let $Y \in \eta(Z)$ and $f \in M(Y)$. We suppose that:

(i) $\left.\theta\right|_{\eta(Z)}$ has the intersection property;

(ii) $f$ is an $(\theta, l)$-contraction.

Then we have that:

(a) $I(f) \cap S(X) \neq \emptyset$;

(b) $F_{f} \neq \emptyset$;

(c) if $F_{f} \in Z$, then $\theta\left(F_{f}\right)=0$.

Theorem 6.10. Let $(X, S(X), M)$ be a f.p.s. on a set $X,(\theta, \eta)$ be a compatible pair with $(X, S(X), M)$. Let $Y \in F_{\eta}$ and $f \in M(Y)$ be such that $f(Y) \in Z$. We suppose that:

(i) $\left.\theta\right|_{\eta(Z)}$ has the intersection property;

(ii) $\left.f\right|_{f(Y)}: f(Y) \rightarrow f(Y)$ is a $(\theta, l)$-contraction.

Then we have that:

(a) $I(f) \cap S(X) \neq \emptyset$;

(b) $F_{f} \neq \emptyset$;

(c) If $F_{f} \in Z$, then $\theta\left(F_{f}\right)=0$.

Our Theorem 6.9 and 6.10 are set-theoretical generalizations of many known fixed point theorems of Darbo type (G. Darbo (1955), J. Appell (2005), J. Banas and K. Goebel (1980), V. Berinde (1997), O. Hadžić (1984), A. Horvat-Marc and M. Berinde (2004), J. Esenfeld - V. Lakshmikantham (1975), I.A. Rus (1983), A. Petruşel (1987), C.S. Barroso - D. O'Regan (2005), R.D. Nussbaum (1969), ...). For example, if $X$ is a Banach space, 
$S(X):=P_{c p, c v}(X), \theta:=\alpha_{K}$ and $f$ is a strong $\left(\alpha_{K}, l\right)$-contraction, then from Theorem 6.9 we have the well known Darbo theorem.

For other results for $(\theta, l)$-contraction, $(\theta, \varphi)$-contractions, for some type of operator in terms of measures of noncompactness, measure of weak noncompactness, measure of nonconvexity, ...see [126], [55], [74], [106], [111], [112], [113], [120], [140], [130], [101], [144], [136], [149], [99], [110], [37], [150], [11], [13], [84], ..

\section{CYCLIC COVERING OF A SET WITH RESPECT TO AN OPERATOR}

Another set-theoretical notion introduced in [124] is the following.

Definition 7.9. Let $X$ be a nonempty set and $f: X \rightarrow X$ be an operator. By definition, a covering (representation in [124]) of $X, X=\bigcup_{i=1}^{m} X_{i}, m \geq 2$, is a cyclic covering of $X$ relative to $f$ iff:

$$
f\left(X_{1}\right) \subset X_{2}, \ldots, f\left(X_{m-1}\right) \subset X_{m}, f\left(X_{m}\right) \subset X_{1} .
$$

In this case we call the operator $f$, a cyclic operator.

The following problem is studied in [124].

Problem XVI. Let $(X, S(X), M)$ be a f.p.s. on a set $X, Y \subset X$ and $f: Y \rightarrow Y$ be an operator. We suppose that $Y=\bigcup_{i=1}^{m} A_{i}$ is a cyclic covering of $Y$ with respect to the operator $f$. The problem is in which conditions we have that:
(a) $A:=\bigcap_{i=1}^{m} A_{i} \neq \emptyset$;
(b) $A \in S(X)$ and $\left.f\right|_{A} \in M(A)$.

If $f$ is a solution of Problem XVI, then $F_{f} \neq \emptyset$.

To study the Problem XVI, the following remarks are useful ([124]).

Inclusion remark. If $X=\bigcup_{i=1}^{m} X_{i}$ is a cyclic covering of $X$ with respect to an operator $f: X \rightarrow X$, then $F_{f} \subset \bigcap_{i=1}^{m} X_{i}$

Fixed point lemma for cyclic operators. Let $(X, S(X), M)$ be a f.p.s. on an L-space $(X, \rightarrow)$. Let $A_{1}, \ldots, A_{m} \in P_{c l}(X), Y:=\bigcup_{i=1}^{m} A_{i}$ and $f: Y \rightarrow Y$ be an operator. We suppose that:

(i) $Y=\bigcup_{i=1}^{m} A_{i}$ is a cyclic covering of $Y$ relative to $f$;

(ii) there exists $x_{0} \in Y$ such that $\left(f^{n}\left(x_{0}\right)\right)_{n \in \mathbb{N}}$ converges;

(iii) if $A:=\bigcap_{i=1}^{m} A_{i} \neq \emptyset$, then $A \in S(X)$ and $\left.f\right|_{A} \in M(A)$.

Then $F_{f} \neq \emptyset$.

Periodic point lemma. Let $(X, S(X), M)$ be a f.p.s. on a set $X$. Let $A_{1}, \ldots, A_{m} \in P(X)$, $Y:=\bigcup_{i=1}^{m} A_{i}$ and $f \in M(Y)$. We suppose that: 
(i) $Y=\bigcup_{i=1}^{m} A_{i}$ is a cyclic representation of $Y$ with respect to the operator $f$;

(ii) at least $A_{i} \in S(X)$;

(iii) the operator $M$ is such that, $g, h \in M \Rightarrow g \circ h \in M$.

Then we have that $F_{f^{m}} \neq \emptyset$. If $F_{f^{m}}=\left\{x^{*}\right\}$, then $F_{f}=\left\{x^{*}\right\}$.

For some results for Problem XVI see, for example, Kirk et al. [75], Păcurar-Rus [86], G. Petruşel [97] and L. Pasicki [87].

\section{EQUIVALENT FIXED POINT EQUATIONS}

8.1. Admissible perturbation of an operator. In this section we shall consider the following problem.

Problem XVII. Let $(X, \rightarrow)$ be an $L$-space and $f: X \rightarrow X$ be an operator. The problem is to find those conditions in which there exists an operator $g: X \rightarrow X$ such that:

(1) $F_{f}=F_{g}$;

(2) $g$ is a weakly Picard operator.

To study this problem, we have introduced in [129] a set-theoretical notion as follows.

Let $X$ be a nonempty set and $G: X \times X \rightarrow X$ be an operator. We suppose that:

$\left(A_{1}\right) G(x, x)=x$, for all $x \in X$;

$\left(A_{2}\right) x, y \in X, G(x, y)=x$ imply $y=x$

Here are some examples of this type of operator $G$.

Example 8.23. Let $(V,+, \mathbb{R})$ be a real vectorial space, $X \subset V$ be a convex subset, $\lambda \in] 0,1[$, $f: X \rightarrow X$ and $G: X \times X \rightarrow X$ be defined by:

$$
G(x, y):=(1-\lambda) x+\lambda y .
$$

Example 8.24. Let $(X, d)$ be a metric space endowed with the $W$-convex structure of Takahashi. The operator $W: X \times X \times[0,1] \rightarrow X$ has the following property:

$$
d(u, W(x, y, \lambda)) \leq \lambda d(u, x)+(1-\lambda) d(u, y) \text {, for all } x, y, u \in X \text { and all } \lambda \in[0,1] .
$$

If in addition we suppose that,

$$
\lambda \in] 0,1[, W(x, y, \lambda)=x \text { implies } y=x,
$$

then we take $G(x, y):=W(x, y, \lambda), \lambda \in] 0,1[$.

Let $f: X \rightarrow X$ be an operator and $G: X \times X \rightarrow X$ be defined as above. We consider the operator $f_{G}: X \rightarrow X$ be defined by

$$
f_{G}(x):=G(x, f(x)), \text { for all } x \in X .
$$

We remark that $F_{f_{G}}=F_{f}$, i.e., the fixed point equations,

$$
x=f(x)
$$

and

are equivalent.

$$
x=f_{G}(x)
$$
$G$.

We call the operator, $f_{G}$, the admissible perturbation of $f$ corresponding to the operator

For $f:(X, \rightarrow) \rightarrow(X, \rightarrow)$ we consider for the fixed point equation,

$$
x=f(x)
$$


the iteration algorithm,

$$
x_{0} \in X, x_{n+1}=G\left(x_{n}, f\left(x_{n}\right)\right), n \in \mathbb{N} .
$$

By definition, this algorithm is convergent if, $x_{n} \rightarrow x^{*}\left(x_{0}\right) \in F_{f}$ as $n \rightarrow \infty$, for all $x_{0} \in X$.

We remark that, $x_{n}=f_{G}^{n}\left(x_{0}\right)$. This implies that our algorithm is convergent iff $f_{G}$ is WPO.

It is clear that, $f_{G}^{\infty}: X \rightarrow F_{f}$ is a set retraction.

To study the Problem XVII it is useful to study the following problems.

Problem XVIII. If $(X, d)$ is a metric space, in which conditions, $f_{G}$ is asymptotically regular?

Problem XIX. If $(X, \rightarrow)$ is an $L$-space, in which conditions, $f_{G}$ is $W P O$ ?

For some results for Problems XVII, XVIII and XIX, see: I.A. Rus [129], [137], V. Berinde [17], [18], V. Berinde - Şt. Măruşter - I.A. Rus [20], V. Berinde - I.A. Rus [23], A. Petruşel I.A. Rus [91], E. Toscano - C. Vetro [158], V. Berinde - A.B. Khan - M. Păcurar [19], C. Ţicală [157], ...

\section{SET RETRACTION AND FIXED POINTS OF NONSELF OPERATORS}

Let us introduce some of the problems of the fixed point theory of nonself operators.

Problem XX (see [130]). Let $(X, S(X), M)$ be a f.p.s. on a set $X, Y \in S(X)$ and $f \in$ $M(Y, X)$. In which conditions we have that, $F_{f} \neq \emptyset$ ?

For a better understanding of this problem we present the following examples.

Example 9.25. [The case of f.p.s. of $R$-contractions] Let $(X, R)$ be a complete $R$-space, $Y \subset X$ be a closed subset and $f: Y \rightarrow X$ be an $R$-contraction. In which conditions we have that $F_{f} \neq \emptyset$ ?

Example 9.26. [The case of f.p.s. of contractions] Let $X$ be a complete metric space, $Y \in$ $P_{c l}(X)$ and $f: Y \rightarrow X$ be a contraction. In which conditions we have that, $F_{f} \neq \emptyset$ ?

Example 9.27. [The case of f.p.s. of Schauder] Let $X$ be a Banach space, $Y \in P_{c p, c v}(X)$ and $f \in C(Y, X)$. In which conditions we have that, $F_{f} \neq \emptyset$ ?

Example 9.28. [The case of f.p.s. of Browder] Let $X$ be a Hilbert space, $Y \in P_{b, c v}(X)$ and $f: Y \rightarrow X$ be a nonexpansive operator. In which conditions we have that, $F_{f} \neq \emptyset$ ?

Problem XXI (see [132]). Let $X$ be a nonempty set, $Y \subset X$ be a nonempty subset of $X$ and $f: Y \rightarrow X$ be a nonself operator. The problem is to find an operator $\rho_{f}: Y \rightarrow Y$ such that $F_{f}=F_{\rho_{f}}$.

To study these problems, we shall use two set-theoretical terms: retraction and retractible operator.

Let $X$ be a nonempty set, $Y \subset X$ be a nonempty subset of $X$. By definition, an operator $\rho: X \rightarrow Y$ is a set retraction if, $\left.\rho\right|_{Y}=1_{Y}$.

If $(X, \leq)$ is an ordered set and $Y \subset X$ is a nonempty subset, then a set retraction $\rho: X \rightarrow Y$ is an ordered set retraction if $\rho$ is an increasing operator. On an ordered set, these are some classes of set retraction. For example, let $\rho: X \rightarrow Y$ be a set retraction. Then:

- $\rho$ is a comparable retraction if $\rho(x)$ is comparable to $x$, for all $x \in X$;

- $\rho$ is progressive retraction (or $u_{p}$-retraction) if $x \leq \rho(x)$, for all $x \in X$;

- $\rho$ is regressive retraction (or down-retraction) if $\rho(x) \leq x$, for all $x \in X$;

- $\rho$ is decreasing retraction if $\rho$ is a decreasing operator. 
If $(X, \tau)$ is a topological space, $Y \subset X$ is a subset of $X$, then a set retraction $\rho: X \rightarrow Y$ is a topological retraction if $\rho$ is continuous.

In a metric space $(X, d)$ we have the following types of retraction: Lipschitz retraction, nonexpansive retraction, continuous retraction, ...

In the paper [28], R.F. Brown introduced the following set-theoretical notion:

Let $\rho: X \rightarrow Y$ be a set retraction and $f: Y \rightarrow X$ be an operator. By definition $f$ is retractible with respect to the retraction $\rho$ if, $F_{f}=F_{\rho \circ f}$. In this case $\rho \circ f$ is called the retract of $f$.

From the above considerations the following problem rises.

Problem XXII. Let $(X, S(X), M)$ be a f.p.s. on a set $X$. The problem is in which conditions for each $Y \in S(X)$ there exists a set retraction, $\rho: X \rightarrow Y$ such that for all $f \in M(Y, X)$, $\rho \circ f \in M(Y)$.

For this problem, we have the following result.

Theorem 9.11 (see Rus [130]). Let $(X, S(X), M)$ be a f.p.s. on a set $X$ and $(\theta, \eta)$ be a compatible pair with $(X, S(X), M)$. Let $Y \in \eta(Z), f: Y \rightarrow X$ be an operator and $\rho: X \rightarrow Y$ be a set retraction. We suppose that:

(i) $\left.\theta\right|_{\eta(Z)}$ is a functional with intersection property;

(ii) $f$ is retractible with respect to $\rho$ and $\rho \circ f \in M(Y)$;

(iii) $\rho$ is $\left(\theta, l_{1}\right)$-Lipschitz $\left(l_{1} \in \mathbb{R}_{+}^{*}\right)$;

(iv) $f$ is a strong $(\theta, l)$-contraction and $l l_{1}<1$.

Then $F_{f} \neq \emptyset$ and if $F_{f} \in Z$, then $\theta\left(F_{f}\right)=0$.

In the case of Schauder f.p.s., from Theorem 9.11 we have:

Theorem 9.12. Let $X$ be a Banach space, $\alpha_{K}: P_{b}(X) \rightarrow \mathbb{R}_{+}$be the Kuratowski measure of noncompactness on $X$ and $f: \bar{B}(0 ; R) \rightarrow X$ be a continuous operator. We suppose that:

(1) $f$ is a strong $\left(\alpha_{K}, l\right)$-conraction;

(2) $f$ is retractible with respect to the radial retraction, $\rho: X \rightarrow \bar{B}(0 ; R)$.

Then $F_{f} \neq \emptyset$ and $F_{f}$ is a compact subset of $X$.

We remark that each of the following conditions implies the condition $(i i)$ in the Theorem 9.12:

(a) (Leray-Schauder). $x \in \partial \bar{B}(0 ; R), f(x)=\lambda x \Rightarrow \lambda \leq 1$;

(b) (Rothe) $f(\partial \bar{B}(0 ; R)) \subset \bar{B}(0 ; R)$;

(c) (Altman) $\|f(x)-x\|^{2} \geq\|f(x)\|^{2}-\|x\|^{2}$, for all $x \in \partial \bar{B}(0 ; R)$.

There are many other boundary conditions which appear in the fixed point theorems for nonself operators (see [55], [114], [126], [140], [132], [72], ...). tor.

In the paper [132] we introduced a set-theoretical notion: generalized retract of an opera-

Let $X$ be a nonempty set, $Y \subset X$ and $f: Y \rightarrow X$ be an operator. By definition, a self operator $\rho_{f}: Y \rightarrow Y$ is called a generalized retract of the nonself operator $f$ iff:

(a) $F_{f}=F_{\rho_{f}}$;

(b) $x \in Y, f(x) \in Y \Rightarrow \rho_{f}(x)=f(x)$.

In [132] we give a generic example of generalized retract of an operator in terms of another set-theoretical notion: interval operator.

Let $X$ be a nonempty set. By definition an operator $[\cdot, \cdot]: X \times X \rightarrow P(X)$ is called interval operator if it satisfies the following conditions:

(a) $[x, y]=[y, x]$, for all $x, y \in X$; 
(b) $x, y \in[x, y]$, for all $x, y \in X$;

(c) $[x, x]=\{x\}$, for all $x \in X$.

Example 9.29. Let $(X, \leq)$ be a supsemilattice and $x, y \in X$. Then

$$
[x, y]_{\leq}:=\{z \in X \mid x \leq z \leq x \vee y\} \cup\{z \in X \mid y \leq z \leq x \vee y\},
$$

defines an interval operator.

Example 9.30. Let $X$ be a linear space and $x, y \in X$. Then

$$
[x, y]_{\leq}:=\{(1-\lambda) x+\lambda y \mid 0 \leq \lambda \leq 1\}
$$

defines an interval operator.

Example 9.31. Let $(X, d)$ be a metric space and $x, y \in X$. Then

$$
[x, y]_{d}:=\{z \in X \mid d(x, z)+d(z, y)=d(x, y)\}
$$

defines an interval operator.

Let $(X,[\cdot, \cdot])$ be a nonempty set with an interval structure. Let $Y \subset X$ and $f: Y \rightarrow X$ be an operator. We suppose that:

$$
x \in Y, f(x) \in X \backslash Y \Rightarrow] x, f(x)] \cap Y \neq \emptyset .
$$

We call this condition, generalized retract condition. Supposing this condition, we define the multivalued operator,

$$
R_{f}: Y \rightarrow P(Y), R_{f}(x):=\left\{\begin{array}{ll}
\{f(x)\} & \text { if } f(x) \in Y \\
] x, f(x)] \cap Y & \text { if } f(x) \in X \backslash Y
\end{array} .\right.
$$

Let $\rho_{f}: Y \rightarrow Y$ be a selection of $R_{f}$, i.e., $\rho_{f}(x) \in R_{f}(x)$, for all $x \in Y$. Then we have that $F_{f}=F_{\rho_{f}}=F_{R_{f}}$. So, $\rho_{f}$ is a generalized retract of $f$.

We have another generic example in the following way.

Let $(X,[\cdot, \cdot])$ be a nonempty set with an interval structure. Let $Y \subset X, f: Y \rightarrow X$ and $x_{0} \in Y$. We suppose that:

$\left.\left.\left(G R_{x_{0}}\right) \quad x \in Y, f(x) \in X \backslash Y \Rightarrow\right] x_{0}, f(x)\right] \cap Y \neq \emptyset$ and $\left.\left.x \in\right] x_{0}, f(x)\right]$.

In the condition $\left(G R_{x_{0}}\right)$, we define the multivalued operator,

$$
R_{f, x_{0}}: Y \rightarrow P(Y), R_{f, x_{0}}(x):=\left\{\begin{array}{ll}
\{f(x)\} & \text { if } f(x) \in Y \\
] x_{0}, f(x)\right] \cap Y & \text { if } f(x) \in X \backslash Y
\end{array} .\right.
$$

Let $\rho_{f, x_{0}}$ be a selection of $R_{f, x_{0}}$. Then:

$$
F_{f}=F_{\rho_{f, x_{0}}}=F_{R_{f, x_{0}}} .
$$

These imply that $\rho_{f, x_{0}}$ is a generalized retract of the nonself operator $f$.

It seems to me that the following set-theoretical notions are important.

Let $X$ be a nonempty set, $Y \subset X, f: Y \rightarrow X$ be a nonself operator and $\rho: X \rightarrow Y$ be a set retraction. By definition, $\partial_{f, \rho}(Y):=\rho(f(Y) \backslash Y)$ is the formal boundary of $Y$ with respect to $f$ and $\rho$.

For other type of formal boundary see F.E. Browder [27], [26], K. Fan [47], R. Precup [99], [100], I.A. Rus [132].

We have the following results.

Lemma 9.1. Let $f: Y \rightarrow X$ be an operator and $\rho: X \rightarrow Y$ be a set retraction. The operator $f$ is retractible with respect to $\rho$ iff,

$$
x \in \partial_{f, \rho}(Y), x=\rho(f(x)) \Rightarrow f(x)=x .
$$


Lemma 9.2. Let $(X, S(X), M)$ be a large f.p.s., $Y \in S(X), \rho: X \rightarrow Y$ be a set retraction and $f: Y \rightarrow X$ be a nonself operator. We suppose that:

(i) $\rho \circ f \in M(Y)$;

(ii) $x \in \partial_{f, \rho}(Y), x=\rho(f(x)) \Rightarrow f(x)=x$.

Then, $F_{f} \neq \emptyset$.

If we take instead of $(X, S(X), M)$, in Lemma 9.2, different examples of large f.p.s. we have fixed point results in structured sets (see [132]). Here are some examples.

Theorem 9.13 (The case of Tarski f.p.s.). Let $(X, \leq)$ be an ordered set with the least element, 0 . Let $Y \in P(X)$ and $f: Y \rightarrow X$ be a nonself operator. We suppose that:

(i) $0 \in Y$;

(ii) $(Y, \leq)$ is a complete lattice;

(iii) $f$ is increasing;

(iv) $f$ satisfies $\left(G R_{x_{0}}\right)$ condition with respect to the order interval $[\cdot, \cdot]_{\leq}$.

Then we have that:

(a) $F_{f} \neq \emptyset$;

(b) $\left(F_{f}, \leq\right)$ is a complete lattice.

Theorem 9.14 (The case of f.p.s. of contractions). Let $(X, d)$ be a complete metric space, $Y \in P_{c l}(X)$ and $f: Y \rightarrow X$ be an operator. We suppose that:

(i) $f$ is an l-contraction;

(ii) $f$ satisfies the (GR) condition with respect to the metric interval, $[\cdot, \cdot]_{d}$.

Then we have that $F_{f}=\left\{x^{*}\right\}$.

The above considerations give rise to the following conjecture.

Problem XXIII (The conjecture of the generalized retracts (Rus [132]). Let $X$ be a Banach space, $Y \subset X$ be a subset with nonempty boundary and $f: Y \rightarrow X$ be a nonself operator. Then, each boundary condition (Leray-Schauder, Rothe, inwardness, outwardness,...) on $f$ implies the existence of a generalized retract of $f$.

References: [121], [126], [140], [29], [50], [55], [106], [130], [35], ...

\section{Multivalued OPERATORS}

For similar problems and results in the fixed point theory of multivalued operators see:

- Set-theoretic notions and problems in the fixed point theory for multivalued operators: [125], [55], [54], [121], [140], [39], ...

- Technique of the fixed point structures for multivalued operators: [118], [126], [140], [153], ...

- Multivalued weakly Picard operators: [142], [89], [140], [90], ...

- Iterative approximation algorithms of fixed point of multivalued operators (admissible perturbations): [91], ...

- Nonself multivalued operators: [126], [55], [54], [74], [140], ...

\section{REFERENCES}

[1] Abian, A., A fixed point theorem for mappings, J. Math. Anal. Appl., 24 (1968), 146-148

[2] Abian, A., A fixed point theorem, Niew Arch. Wisk., 16 (1968), 184-185

[3] Agratini, O. and Rus, I. A., Iterates of some bivariate approximation process via weakly Picard operators, Nonlinear Anal. Forum, 8 (2003), No. 2, 158-168

[4] Aldea, F., Surjectivity via fixed point structure, Seminar on Fixed Point Theory, Babeş-Bolyai Univ., ClujNapoca, 1997, 9-14 
[5] András, S. and Rus, I. A., Iterates of Cesàro operators, via fixed point principles, Fixed Point Theory, 11 (2010), No. 2, 171-178

[6] Andres, J., Some standard fixed point theorems revisited, Atti. Sem. Mat. Fiz. Univ. Modena, 49 (2001), 455-471

[7] Andres, J. and Fišer, J., Multivalued fractals, Chaos, solitons and fractals, 24 (2005), No. 3, 665-700

[8] Angelov, V. G., A converse to a contraction mapping theorem in uniform spaces, Nonlinear Anal., 12 (1988), 989-996

[9] Anisiu, M. C., Fiexd point theorems for retractible mappings, Seminar on Functional Anal. and Numerical Methods, Babeş-Bolyai Univ., Cluj-Napoca, 1989, 1-10

[10] Anisiu, M. C. and Anisiu, V., On the closedness of sets with the fixed point property for contractions, Revue d'Anal. Numér. Théorie l'Approx., 26 (1997), No. 1-2, 13-17

[11] Appell, J., Measures of noncompactness, condensing operators and fixed points: an application-oriented survey, Fixed Point Theory, 6 (2005), 157-229

[12] Bacoţiu, C., Picard Operators and Applications, Ph. D. Dissertation, Babeş-Bolyai Univ., Cluj-Napoca, 2004

[13] Ban, A. I. and Gal, S. G., Defects of Properties in Mathematics; Quantitative Characterizations, World Scientific, 2002

[14] Barr, M. and Wells, C., Cathegory for Computing Science, Prentice Hall, New York, 1990

[15] Bege, A., Fixed points of R-contractions, Studia Univ. Babeş-Bolyai, Math., 47 (2002), No. 4, 19-25

[16] Bellen, A. and Valčič, A., Non-cyclic transformations and uniform convergence of the Picard sequences, Rend. Ist. di Matem. Univ. Trieste, 4 (1972), fas. 1, 1972

[17] Berinde, V., Iterative Approximation of Fixed Points, Springer, 2007

[18] Berinde, V., Convergence theorems for admissible perturbations of $\varphi$-pseudocontractive operators, Miskolc Math. Notes, 15 (2014), 27-37

[19] Berinde, V., Khan, A. R. and Păcurar, M., Convergence theorems for admissible perturbations of pseudocontractive operators, Miskolc Math. Notes, 15 (2014), No. 1, 27-37

[20] Berinde, V., Măruşter, Şt. and Rus, I. A., An abstract point of view on iterative approximation of fixed points of nonself operators, J. Nonlinear Convex Anal., 15 (2014), No. 5, 851-865

[21] Berinde, V., Păcurar, M. and Rus, I. A., From a Dieudonné theorem concerning Cauchy problem to an open problem in the theory of weakly Picard operators, Carpathian J. Math., 30 (2014), No. 3, 283-292

[22] Berinde, V., Petruşel, A., Rus, I. A. and Şerban, M.-A., The retraction-displacement condition in the theory of fixed point equation with a convergence iterative algorithm, 75-106. In: T.M. Rassias and V. Gupta (eds.), Mathematical Analysis, Approximation Theory and Their Application, Springer, 2016.

[23] Berinde, V. and Rus, I. A., Asymptotic regularity, fixed points and successive approximations, Filomat, 34:3 (2020), 965-981

[24] Blass, A., The interaction between category theory and set theory, Contemporary Math., 30 (1984), 5-29

[25] Bourbaki, N., Théorie des ensembles, Herman, Paris, 1956

[26] Browder, F. E., A new generalization of the Schauder fixed point theorem, Math. Ann., 174 (1967), 285-290

[27] Browder, F. E., Continuation of solutions of equations under homotopies of single-valued and multivalued mappings, 13-22. In: S. Swaminathan (ed.), Fixed Point Theory and its Applications, Academic Press, 1976

[28] Brown, R. F., Retraction mapping principle in Nielsen fixed point theory, Pacific J. Math., 115 (1984), 277-297

[29] Buică, A., Principii de coincidenţ̧̆ şi aplicaţii, Presa Univ. Clujeană, Cluj-Napoca, 2001

[30] Buică, A., Rus, I. A. and Şerban, M.-A., Zero point principle of ball-near identity operators and applications to implicit operator problem, Fixed Point Theory, 21 (2020), No. 1, 79-92

[31] Bunlue, N. and Suantui, S., Convergence theorems of fixed point iterative methods defined by admissible functions, Thai J. Math., 13 (2015), No. 3, 527-537

[32] Cătinaş, T. and Otrocol, D., Iterates of multivalued Cheney-Sharma operators, J. Comput. Anal. Appl., 15(2013), No. $7,1240-1246$

[33] Cătinaş, T., Otrocol, D. and Rus, I. A., The iterates of positive linear operator with the set of constant functions as the fixed point set, Carpathian J. Math., 32 (2016), No. 2, 165-172

[34] Chidume, C. E., Geometric Properties of Banach Spaces and Nonlinear Iterations, Springer, 2009

[35] Chiş-Novac, A., Precup, R. and Rus, I. A., Data dependence of fixed points for nonself generalized contractions, Fixed Point Theory, 10 (2009), No. 1, 97-104

[36] Chu, S. C. and Diaz, J. B., Remarks on a generalization of Banach's principle of contraction mappings, J. Math. Anal. Appl., 11 (1965), 440-446

[37] Coroian, L., $d_{D P}$-condensing mappings and Picard mappings, Seminar on Fixed Point Theory, Babeş-Bolyai Univ., Cluj-Napoca, 1988, 17-22

[38] Cristescu, R., Ordered Vector Spaces and Linear Operators, Abacus Press, 1976

[39] De Blasi, F. S., Semifixed sets of maps in hyperspaces with applications to set differential equations, Set-Valued Anal., 14 (2006), 263-272

[40] Deaconescu, M., The fixed point set for injective mappings, Studia Univ. Babeş-Bolyai, Math., 29 (1984), 11-15 
[41] Dincuță, V., An application of the weakly Picard operators techniques to a Dirichlet problem, Seminar on Fixed Point Theory, Cluj-Napoca, 1 (2000), 35-38

[42] Domenach, F. A., Structures latticielles, correspondances de Galois contraintes et classification symbolique, Université Paris I, 2002, HAL archives-ouverts

[43] Dudley, R. M., On sequential convergence, Trans. Amer. Math. Soc., 112(1964), 483-507. Corrections: 148 (1970), 623-624

[44] Duffus, D., Poguntke, W. and Rival, I., Retracts and the fixed point problem for finite partially ordered sets, Canad. Math. Bull., 23 (1980), 231-236

[45] Duffus, D. and Rival, I., Retract of partially ordered sets, J. Austral. Math. Soc., 27 (1979), 495-506

[46] Espinola, R., López, G. and Petruşel, A., Crossed cartezian product of multivalued operators, Nonlinear Funct. Anal. Appl., 12 (2007), 563-575

[47] Fan, K., Extensions of two fixed point theorems of F.E. Browder, Math. Z., 112 (1969), 234-290

[48] Farmakis, I. and Moskowitz, M., Fixed Point Theorems and Applications, World Scientific, 2013

[49] Fréchet, M., Les espaces abstraits, Gauthier-Villars, Paris, 1928

[50] Goebel, K., Problems I left behind, Proceed. of the $10^{\text {th }}$ IC-FPTA, 9-20, 2013

[51] Goebel, K. and Sedlak, E., Nonexpansive retractions in Hilbert spaces, Ann. Univ. M. Curie-Sklodowska, Lublin, sec. A, 63 (2009), 83-90

[52] Gonska, H., Kacsó, D. and Piţul, P., The degree of convergence of over-iterated positive linear operators, J. Appl. Funct. Anal., 1 (2006), 403-423

[53] Gonska, H. and Piţul, P., Remarks on an article of J.P. King, Comment. Math. Univ. Carolinae, 46(2005), No. 4, 645-652

[54] Górniewicz, L., Topological fixed point theory of multivalued mappings, Kluwer, 1999

[55] Granas, A. and Dugundji, J., Fixed Point Theory, Springer, 2003

[56] Grudzindski, W., On the discrete Banach principle, Zesz. Nauk. Politech. Lódz, Mat., 695 (1994), 81-88

[57] Grundmane, D., Fixed point for nonself mappings in metric spaces, Mathematica Moravica, 9 (2005), 5-11

[58] Halmos, P. R., Naive Set Theory, D. von Nostrand Co., Princeton, 1960

[59] Heitzig, J., Many familiar categories can be interpreted as categories of generalized metric spaces, Applied Categorial Structures, 10 (2002), 505-530

[60] Horvat-Marc, A., Retraction methods in fixed point theory, Seminar on Fixed Point Theory Cluj-Napoca, 1 (2000), 39-54

[61] Horvat-Marc, A. and Berinde, M., Another general fixed point principles, Carpathian J. Math., 20 (2004), 45-49

[62] Hu, S., Theory of Retracts, Wayne State Univ., 1965

[63] Istrăţescu, V. I., Fixed Point Theory, D. Reidel, 1981

[64] Jachymski, J., Fixed point theorems in metric and uniform spaces via the Knaster-Tarski principle, Nonlinear Anal., 32 (1998), No. 2, 225-233

[65] Jachymski, J., Converses to fixed point theorems of Zermelo and Caristi, Nonlinear Anal., 52 (2003), 1455-1463

[66] Jachymski, J., A discrete fixed point theorem of Eilenberg as a particular case of the contraction principle, Fixed Point Theory Appl., 2004:1 (2004), 31-36

[67] Jachymski, J., Convergence of iterates of linear operators and the Kelisky-Rivlin type theorems, Studia Math., 195 (2009), No. 2, 99-112

[68] Jawhari, E. M., Misane, D. and Pauzet, M., Retracts: graphs and ordered sets from the metric point of view, Contemporary Math., 57 (1986), 175-226

[69] Kelley, J. L., General Topology, von Nostrand, 1955

[70] Khamsi, M. A. and Kirk, W. A., An Introduction to Metric Spaces and Fixed Point Theory, John Wiley and Sons, 2001

[71] Kirk, W. A., Metric fixed point theory: a brief retrospective, Fixed Point Theory Appl., (2015), 2015:215

[72] Kirk, W. A. and Morales, C. H., Nonexpansive mappings: boundary/inwardness conditions and localy theory, 299-321, in: W. A. Kirk and B. Sims (eds.), Handbook of Metric Fixed Point Theory, Kluwer, 2001

[73] Kirk, W. A. and Shahzad, N., Fixed Point Theory in Distance Spaces, Springer, 2014

[74] Kirk, W. A. and Sims, B. (Eds.), Handbook of Metric Fixed Point Theory, Kluwer, 2001

[75] Kirk, W. A., Srinivasan, P. S. and Veeramani, P., Fixed points for mappings satisfying cyclical contractive conditions, Fixed Point Theory, 4 (2003), No. 1, 79-89

[76] Kuratowski, K., Topology, Academic Press, New York, 1966

[77] Lawvere, F. W., Diagonal arguments and cartesian closed categories, Theory and Appl. of Category, 15 (2006), 1-13

[78] Lemmert, R. and Volkmann, P., Un théorème de point fixe dans les ensembles, Mathematica, 31 (1989), 69-73

[79] Lim, T.-C., Remarks on some fixed point theorems, Proc. Amer. Math. Soc., 60 (1976), 179-182

[80] Mac Lane, S., Categories for the Working Mathematiciens, Springer, 1971

[81] Mac Lane, S., Mathematics: Form and Function, Springer, 1986 
[82] Monna, A. F., Algebraic and set-theoretic aspects of the evolution of mathematics, Indagationes Math., 45 (1983), fas. 3, 329-341

[83] Mureşan, A. S., Mappings of Picard, Bessage and Janos type, Bul. Ştiinţ. Univ. Baia Mare, ser. B, Matem-Inform., 12 (1996), 85-90

[84] Nussbaum, R. D., The fixed point index and fixed point theorems for $K$-set-contractions, Ph. D. Dissertation, Univ. of Chicago, 1969

[85] Opoitsev, V. I., A converse to the principle of contraction maps, Russian Math. Surveys, 31 (1976), No. 4, 175-204

[86] Păcurar, M. and Rus, I. A., Fixed point theory for cyclic $\varphi$-contractions, Nonlinear Anal., 72 (2010), No. 3-4, 1181-1187

[87] Pasicki, L., Dislocated quasi-metric and generalized contractions, Fixed Point Theory, 19 (2018), No. 1, 359-368

[88] Petruşel, A., On the fixed points set for some contractive multivalued operators, Mathematica, 42 (2000), No. 2, 179-186

[89] Petruşel, A., Multivalued weakly Picard operators and applications, Sci. Math. Jpn., 59 (2004), 167-202

[90] Petruşel, A. and Rus, I. A., Multivalued Picard and weakly Picard operators, Proc. IC-Fixed Point Theory (Valencia, 2003), 207-226, Yokohama Publ., 2004

[91] Petruşel, A. and Rus, I. A., An abstract point of view on iterative approximation schemes of fixed points for multivalued operators, J. Nonlinear Sci. Appl., 6 (2013), No. 2, 97-107

[92] Petruşel, A. and Rus, I. A., Graphic contraction principle and applications, 395-416. In: Mathematical Analysis and Applications, Th. M. Rassias et al.(eds.), Springer, 2019

[93] Petruşel, A. and Rus, I. A., The relevance of a metric condition on a pair of operators in common fixed point theory, In: Y. J. Cho et al. (eds.), Metric Fixed Point Theory and Applications, Springer, 2021

[94] Petruşel, A., Rus, I. A. and Şerban, M. A., The role of equivalent metrics in fixed point theory, Topological Methods in Nonlinear Anal., 41 (2013), No. 1, 85-112

[95] Petruşel, A., Rus, I. A. and Şerban, M.-A., Nonexpansive operators as graphic contractions, J. Nonlinear Convex Anal., 17 (2016), No. 7, 1409-1415

[96] Petruşel, A., Rus, I. A. and Şerban, M.-A., Fixed point structures, invariant operators, invariant partitions, and applications to Carathéodory integral equations, 497-515. In: P. M. Pardalos and T. M. Rassias (eds.), Contributions in Mathematics and Engineering, Springer, 2016

[97] Petruşel, G., Cyclic representations and periodic points, Stud. Univ. Babeş-Bolyai, Math., 50 (2005), 107-112

[98] Precup, R., Sur l'axiomatique des éspaces à convexité, Rev. Anal. Numér. Théor. Approx., 9 (1980), No. 2, 95-103

[99] Precup, R., Generalized topological transversality and existence theorems, Libertas Mathematica, 11 (1991), 65-79

[100] Precup, R., Existence theorems for nonlinear problems by continuation methods, Nonlinear Anal., 30 (1997), No. 6, 3313-3322

[101] Precup, R. and Rus, I. A., Some fixed point theorems in terms of two measures of noncompactness, Mathematica, 56 (2014), No. 2, 158-165

[102] Rus, I. A., Asupra existenţei punctelor fixe ale unei aplicaţii, Lucrări Ştiinţifice, Inst. Pedagogic Tîrgu-Mureş, 2 (1970), 21-23

[103] Rus, I. A., Teoria punctului fix în structuri algebrice, Univ. Babeş-Bolyai, Cluj, 1971

[104] Rus, I. A., Quelques remarques sur la théorie de point fixe, Studia Univ. Babeş-Bolyai, fas. 2, 1971, 5-7; 1972, fas. 2, 5-7; 1973, 3-5

[105] Rus, I. A., Teoria punctului fix în analiza funcţională, Univ. Babeş-Bolyai, Cluj-Napoca, 1973

[106] Rus, I. A., Principii şi aplicaţii ale teoriei punctului fix, Editura Dacia, Cluj-Napoca, 1979

[107] Rus, I. A., Metrical Fixed Point Theorems, Univ. Babeş-Bolyai, Cluj-Napoca, 1979

[108] Rus, I. A., Punct de vedere categorial în teoria punctului fix, Seminarul Itinerant, Timişoara, 1980, 205-209

[109] Rus, I. A., Generalized Contractions, Seminar on Fixed Point Theory, Babeş-Bolyai Univ., 1983, 1-130

[110] Rus, I. A., On a theorem of Eisenfeld-Lakshmikantham, Nonlinear Anal., 7 (1983), 279-281

[111] Rus, I. A., Fixed point structures, Mathematica, 28 (1986), No. 1, 59-64

[112] Rus, I. A., Further remarks on the fixed point structures, Studia Univ. Babeş-Bolyai, 31 (1986), No. 4, 41-43

[113] Rus, I. A., Technique of the fixed point structures, Seminar on Fixed Point Theory, 1987, 3-16

[114] Rus, I. A., Retraction method in the fixed point theory in ordered structures, Seminar on Fixed Point Theory, 1988, 1-8

[115] Rus, I. A., Discrete fixed point theorems, Studia Univ. Babeş-Bolyai, 33 (1988), No. 3, 61-64

[116] Rus, I. A., R-contractions, Studia Babeş-Bolyai, 34 (1989), No. 3, 58-62

[117] Rus, I. A., Some remarks on coincidence theory, Pure Math. Manuscript, 9 (1990-91), 137-148

[118] Rus, I. A., Technique of the fixed point structures for multivalued mappings, Math. Jpn., 38(1993), No. 2, 289-296

[119] Rus, I. A., Weakly Picard mappings, Comment. Math. Univ. Carolinae, 34,4 (1993), 769-773

[120] Rus, I. A., Some open problems in fixed point theory by means of fixed point structures, Libertas Math., 14 (1994), 65-84

[121] Rus, I. A., Generalized Contractions and Applications, Cluj Univ. Press, Cluj-Napoca, 2001

[122] Rus, I. A., Picard operators and applications, Sci. Math. Jpn., 58 (2003), No. 1, 191-219 
[123] Rus, I. A., Iterates of Bernstein operators, via contraction principle, J. Math. Anal. Appl., 292 (2004), $259-261$

[124] Rus, I. A., Cyclic reprezentations and fixed points, Ann. T. Popoviciu Seminar Funct. Eq. Approximation and Convexity, 3 (2005), 171-178

[125] Rus, I. A., Set-theoretic aspects of fixed point theory of multivalued operators: open problems, Sci. Math. Jpn., 62 (2005), No. 2, Notices from the ISMS, 6pp.

[126] Rus, I. A., Fixed Point Structure Theory, Cluj Univ. Press, Cluj-Napoca, 2006.

[127] Rus, I. A., Fixed point and interpolation point set of a positive linear operator on $C(\bar{D})$, Studia Univ. BabeşBolyai, Math., 55 (2010), No. 4, 243-248

[128] Rus, I. A., Some nonlinear functional differential and integral equations, via weakly Picard operator theory: a survey, Carpathian J. Math., 26 (2010), No. 2, 230-258

[129] Rus, I. A., An abstract point of view on iterative approximation of fixed points: impact on the theory of fixed point equations, Fixed Point Theory, 13 (2012), No. 1, 179-192

[130] Rus, I. A., Five open problems in fixed point theory in terms of fixed point structures (I): singlevalued operators, Proc. of the 10 ${ }^{\text {th }}$ IC-FPTA 2012 (2013), 39-60

[131] Rus, I. A., Heuristic introduction to weakly Picard operator theory, Creat. Math. Inform., 23 (2014), No. 2, 243-252

[132] Rus, I. A., The generalized retraction methods in fixed point theory for nonself operators, Fixed Point Theory, 15 (2014), No. 2, 559-578

[133] Rus, I. A., Iterates of increasing linear operators, via Maia's fixed point theorem, Studia Univ. Babeş-Bolyai Math., 60 (2015), No. 1, 91-98

[134] Rus, I. A., Relevant classes of weakly Picard operators, Analele Univ. Vest Timişoara, Math.-Inform., 54 (2016), No. 2, 3-19

[135] Rus, I. A., Some variants of contraction principle, generalizations and applications, Studia Univ. Babeş-Bolyai, Math., 61 2016), No. 3, 343-358

[136] Rus, I. A., Some problems in the fixed point theory, Adv. Theory Nonlinear Anal. Appl., 2 (2018), No. 1, 1-10

[137] Rus, I. A., Convergence results for fixed point iterative algorithms in metric spaces, Carpathian J. Math., 35 (2019), No. 2, 209-220

[138] Rus, I. A. and Mureşan, A. S., Examples and counterexamples for Janos mappings, Seminar on Fixed Point Theory, Babeş-Bolyai Univ., Cluj-Napoca, 1984, 63-66

[139] Rus, I. A., Mureşan, S. and Miklós, E., Maximal fixed point structures, Studia Univ. Babeş-Bolyai, Math., 48 (2003), No. 3, 141-145

[140] Rus, I. A., Petruşel, A. and Petruşel, G., Fixed Point Theory, Cluj Univ. Press, Cluj-Napoca, 2008

[141] Rus, I. A., Petruşel, A. and Şerban, M.-A., Weakly Picard operators: equivalent definitions, applications and open problems, Fixed Point Theory, 7 (2006), No. 1, 3-22

[142] Rus, I. A., Petruşel, A. and Sîntămărian, A., Data dependence of the fixed points set of some multivalued weakly Picard operators, Nonlinear Anal., 52 (2003), 1947-1959

[143] Rus, I. A. and Şerban, M.-A., Basic problems of the metric fixed point theory and the relevance of a metric fixed point theorem, Carpathian J. Math., 29 (2013), No. 2, 239-258

[144] Rus, I. A. and Şerban, M.-A., Some fixed point theorems on cartesian product in terms of vectorial measure of noncompactness, Studia Univ. Babeş-Bolyai, 59 (2014), No. 1, 103-111

[145] Rus, I. A. and Şerban, M.-A., Some fixed point theorems for nonself generalized contraction, Miskolc Math. Notes, 17 (2016), No. 2, 1021-1031

[146] Sato-Andrade, J. and Varela, F. J., Self-references and fixed points: a discussion and an extension of Lawvere's theorem, Acta Appl. Math., 2 (1984), 1-19

[147] Schröder B. S. W., On retractable sets and the fixed point property, Algebra Universală, 33 (1995), 149-158

[148] Schröder, B. S. W., Uniqueness of the core for chain-complete ordered sets, Preprint, Hampton Univ., 1996

[149] Șerban, M.-A., Technique of fixed point structure for the mappings on product spaces, Seminar on Fixed Point Theory, Babeş-Bolyai Univ., 1998, 1-18

[150] Şerban, M.-A., Teoria punctului fix pentru operatori definiţi pe produs cartezian, Presa Universitară Clujeană, Cluj-Napoca, 2001

[151] Şerban, M.-A., Fibre contraction principle with respect to an iterative algorithm, J. of Operators, Vol. 2013, Article ID 408791, 6 pp.

[152] Singer, I., Abstract Convex Analysis, John Wiley and Sons, Toronto, 1997

[153] Sîntămărian, A., Contribuţii la studiul structurilor de punct fix pentru operatori multivoci, Ph.D. Dissertation, Babeş-Bolyai University, Cluj-Napoca, 2001

[154] Stein, J. D., A systematic generalization procedure for fixed point theorems, Rocky Mountain J. Math., 30 (2000), No. 2, 735-754

[155] Suzuki, T., Some notes on Bauschke's condition, Nonlinear Anal., 67 (2007), 2224-2231 
[156] Thompson, R. B., A metatheorem for fixed point theories, Comment. Math. Univ. Carolinae, 11 (1970), No. 4, 813-815

[157] Tiicală, C., Approximating fixed points of asymptotically demicontractive mappings by iterative schemes defined as admissible perturbations, Carpathian J. Math., 33 (2017), No. 3, 381-388

[158] Toscano, E. and Vetro, C., Fixed point iterative schemes for variational inequality problems, J. Convex Anal., 25 (2018), No. 2, 701-715

[159] Wisniewski, K., On functions without fixed points, Ann. Soc. Math. Pol., 17 (1973), 227-228

DEPARTMENT OF MATHEMATICS

BABEŞ-BOlyai University, FACUlty OF MATHEMATICS AND COMPUTER SCIENCE

Mihail Kogalniceanu Street, No. 1, 400084, Cluj-Napoca, Romania

E-mail address: iarus@math. ubbcluj.ro 